however, further exploration of this area is needed before definitive conclusions can be drawn.

\section{Tamoxifen - a potential treatment for women in the manic phase of bipolar affective disorder?}

\section{J Kulkarni, C Gurvich, H Gilbert, R Anderson, S Sheppard, K Garland, A de Castella, P Fitzgerald}

Alfred Psychiatry Research Centre, Melbourne, Australia

Background: Bipolar affective disorder (BPAD) is an illness with high morbidity and mortality. Lithium and other anticonvulsant drugs are the main treatments for BPAD, despite little being known about their mechanisms of action. Recent attempts to elucidate the biochemical actions of these drugs have focused on the protein kinase C (PKC) pathways. Another PKC inhibitor hypothesized to be effective in the treatment of mania is tamoxifen, a synthetic nonsteroidal antiestrogen. The aim of the current study was to test and compare the effectiveness of two adjunctive antiestrogen agents (tamoxifen or progesterone) in the treatment of acute mania.

Methods: A 28-day, three-arm (40 mg/day oral tamoxifen or $20 \mathrm{mg}$ /day oral progesterone or oral placebo), double-blind, placebo-controlled, adjunctive study of 34 women with mania was conducted. All patients also received a mood stabilizer as the baseline treatment. Manic symptoms and psychopathology were measured weekly using the CARS-M and Positive and Negative Syndrome Scale rating scales together with estrogen, progesterone and gonadotropin levels. Cognitive functioning (RBANS) was assessed in a subsample of five participants at baseline and repeated on day 28 .

Results: Results indicated a decline in the symptoms of mania and psychopathology in the tamoxifen group, and to a lesser extent in the progesterone and control groups. The tamoxifen group also had significant changes in estrogen levels, as well as correlations between estrogen and mania ratings.

Conclusion: The results suggest that tamoxifen may be a useful adjunct in the treatment of acute manic symptoms in women with BPAD.

\section{The use of selective estrogen receptor modulators in the treatment of menopausal women with schizophrenia}

\section{J Kulkarni, H Gilbert, C Gurvich, A de Castella, $P$ Fitzgerald, $S$ Davis}

Alfred Psychiatry Research Centre, Melbourne, Australia
Background: Estrogen modulates rat brain dopamine and serotonin systems in a way that mimics atypical antipsychotics. Our work indicates that adjunctive estrogen is a useful treatment in women of childbearing age with schizophrenia. We studied the use of a selective estrogen receptor modulator (SERM) in menopausal women with schizophrenia.

Aim: To test and compare the effects of adjunctive use of an SERM (raloxifene) and standard hormone therapy (HT) on psychotic symptoms in menopausal women with schizophrenia. To examine the effect of an SERM and HT on cognition in menopausal women with schizophrenia.

Method: A double-blind, 3-month, placebo-controlled, adjunctive study of raloxifene $(60 \mathrm{mg} /$ day $)$ vs. HT (2 mg estradiol plus $10 \mathrm{mg}$ dihydroprogesterone) vs. placebo was conducted. Participants received standardized doses of risperidone (or equivalent doses of similar antipsychotic medication). Psychopathology was measured fortnightly using the Positive and Negative Syndrome Scale rating scale. Cognitive testing and sex hormone assays were conducted monthly.

Results: Data collected from 23 participants indicated that while SERM or HT adjuncts did not result in an improvement in psychotic symptoms when compared with risperidone alone, the use of adjunctive SERM resulted in improved cognitive performance on working and verbal memory tasks when compared with the HT or risperidone alone.

Conclusions: The use of adjunctive SERM at $60 \mathrm{mg} /$ day may induce a mild increase in cognitive performance in menopausal women with schizophrenia. Yaffe et al. (2005) show that $120 \mathrm{mg} /$ day raloxifene was more effective in improving cognition in healthy postmenopausal women. We are undertaking a new study with this increased dose of raloxifene.

\section{The estrogen 100}

\section{J Kulkarni, S Sheppard, S White, C Bartholomeusz, C Gurvich, A de Castella, P Fitzgerald}

Alfred Psychiatry Research Centre, Melbourne, Australia

Aim: To compare the efficacy of adjunctive transdermal estradiol with adjunctive placebo in the treatment of acute psychotic symptoms in 100 women with schizophrenia.

Background: Estrogen has been shown in animal studies to have dopamine downregulation effects and has also been shown to impact the serotonergic system. Additionally, there are clinical case reports of women whose schizophrenic symptomatology is exacerbated at low estrogen phases of the menstrual cycle. Similarly, there are clinical case reports of women with 